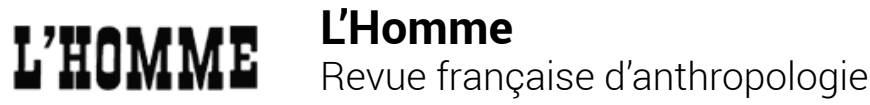

225 | 2018

Varia

\section{Pierre Bitoun \& Yves Dupont, Le Sacrifice des paysans. Une catastrophe sociale et anthropologique}

\section{Léo Magnin}

\section{(2) OpenEdition \\ 1 Journals}

\section{Édition électronique}

URL : http://journals.openedition.org/lhomme/30839

DOI : 10.4000/lhomme.30839

ISSN : 1953-8103

Éditeur

Éditions de l'EHESS

\section{Édition imprimée}

Date de publication : 15 mars 2018

Pagination : 214-216

ISBN : 978-2-7132-2733-2

ISSN : 0439-4216

Référence électronique

Léo Magnin, «Pierre Bitoun \& Yves Dupont, Le Sacrifice des paysans. Une catastrophe sociale et anthropologique », L'Homme [En ligne], 225 | 2018, mis en ligne le 15 mars 2018, consulté le 06 janvier 2021. URL : http://journals.openedition.org/lhomme/30839; DOI : https://doi.org/10.4000//homme. 30839

Ce document a été généré automatiquement le 6 janvier 2021.

(c) École des hautes études en sciences sociales 


\title{
Pierre Bitoun \& Yves Dupont, Le Sacrifice des paysans. Une catastrophe sociale et anthropologique
}

\author{
Léo Magnin
}

\section{RÉFÉRENCE}

Pierre Bitoun \& Yves Dupont, Le Sacrifice des paysans. Une catastrophe sociale et anthropologique. Paris, L’Échappée, 2016, 331 p. (« Pour en finir avec »).

1 Après avoir signé avec Pierre Alphandéry Les Champs du départ ${ }^{1}$, livre qui condensait une quinzaine d'années de recherches historiques et sociologiques sur l'agriculture française, puis L'Équivoque écologique ${ }^{2}$, qui retraçait les dévoiements autoritaires et techniques de l'écologie politique, Pierre Bitoun et Yves Dupont présentent à leurs lecteurs Le Sacrifice des paysans. Une catastrophe sociale et anthropologique. Cet ouvrage résume et prolonge leurs travaux sur le monde paysan en les resituant dans une perspective historique au long cours, afin d'en dégager des clés de lecture socioanthropologiques. L'essai opère une double synthèse. Il ponctue d'abord l'aboutissement des carrières scientifiques des deux auteurs, marquées par leur engagement aux côtés de la Confédération paysanne, syndicat agricole situé à gauche de l'échiquier politique. Ensuite, cette synthèse biographique autorisant une synthèse théorique, la question suivante est posée: quels sont les mouvements de fond anthropologiques à même de rendre compte de l'élimination sociale des paysans?

Pierre Bitoun et Yves Dupont répondront à cette question en deux parties. Dans la première, intitulée «Des Trente Glorieuses aux Quarante Honteuses », ils brossent un tableau de la modernité française de la seconde moitié du $\mathrm{xx}^{\mathrm{e}}$ siècle, en exposant les processus qui ont conduit à la promotion d'une société de "troisième voie ", entre l'Ouest et l'Est, faite d'une révolution des mœurs, de productivisme et de quantification des activités humaines. À l'intérieur de ce panorama sociohistorique, la seconde partie, 
"Projet prométhéen et ensauvagement des paysans », leur permet d'introduire le cas particulier de la paysannerie, en s'appuyant sur le concept d'ethnocide.

3 L'originalité de l'ouvrage, dont le titre est sans ambiguïtés dédié à la question paysanne, tient de prime abord au peu de pages strictement consacrées à celle-ci. La méthode choisie consiste à suivre le fil paysan au sein des transformations qui affectent le monde social dans son entièreté, afin de mieux appréhender à la fois la disparition des paysans et l'élan historique qui l'a portée. Il s'agit, en définitive, de comprendre réciproquement ce qui fait disparaître et ce qui disparaît - la société industrielle et la civilisation paysanne -, en actualisant les acquis des classiques de la sociologie rurale axés sur la "fin des paysans »". Pour ce faire, les auteurs se mettent en quête de «la spécificité de ce bouc émissaire des Temps modernes » (p. 35), capable d'éclairer une réalité plus vaste : «la question centrale [...], le sacrifice des paysans [...] est aussi, on l'aura désormais compris, le sacrifice de tous les autres ou presque » (p. 38).

L'histoire des Trente Glorieuses (1945-1975) est abordée selon le point de vue d'«héritiers critiques» (p. 48). Au sortir de la guerre, le Conseil national de la Résistance amorce un rapprochement entre des sensibilités religieuses et politiques aussi éloignées que le catholicisme et l'athéisme, le communisme et le libéralisme. Ce compromis défend le modèle d'une société de "troisième voie ", entre la démocratie parlementaire de la III ${ }^{\mathrm{e}}$ République et les régimes totalitaires, tout en conférant à l'État un rôle central dans sa mise en œuvre. Cette mise en œuvre est confiée à une minorité agissante, qui est au service du public dans une intention progressiste et organiciste, et s'apparente à une technocratie disposant de son école, l'École nationale d'administration (ENA), dès 1945, et d'une puissante institution politique, le Commissariat général au Plan, en 1946.

5 Cette période constitue le creuset d'un ethos syncrétique (p. 83), où se mêlent la doctrine de la Jeunesse agricole catholique, le personnalisme d'Emmanuel Mounier, l'existentialisme de Jean-Paul Sartre et les espoirs du marxisme. Leur dénominateur commun, qui réside dans un élan transformateur et une confiance en l'avenir, a accéléré l'homogénéisation sociale à l'origine de la classe moyenne et favorisé le développement du monde associatif, donc, la voie d'une «socio-économie duale» (p. 91) - distinguant, d'un côté, ceux qui peuvent composer avec les chocs du marché et, de l'autre, ceux qui les subissent. Il a aussi entériné un mode d'ascension sociale, dont le moteur est l'éducation nationale et populaire, dépositaire de la mission civilisatrice. Il a enfin eu deux héritages politiques : le progressisme technique, incarné par la fondation Saint-Simon, et l'écologie politique, qui s'inscrit en critique de la modernité.

6 La marche du progrès économique est, pour les auteurs, toujours doublée d'une débauche de moyens techniques. Leur analyse du processus de modernisation se fonde sur la thèse de la superfluité de l'homme face aux objets qu'il produit, dont ils trouvent la première formulation dans les Manuscrits de 1844 de Karl Marx ${ }^{4}$, reprise ensuite par Hannah Arendt et Gunther Anders. Les agents de cette dépréciation de l'humain ont en commun de chiffrer le réel : statistiques d'État, puissance des normes et des standards, gouvernement et autogouvernement via les indicateurs de performance ${ }^{5}$. La réalité du monde paysan ne saurait donc être énoncée, pour être comprise, que sous la forme d'un nombre décroissant. Alors que ses membres étaient 7,4 millions en 1946, ils ne sont plus que 850000 en 2013, passant ainsi de $36 \%$ à $3 \%$ de la population active française.

7 Dans la seconde partie de l'ouvrage, Pierre Bitoun et Yves Dupont s'attachent à montrer que les politiques de développement ont été, dans les faits, des politiques de 
dépaysannisation. Si le progrès s'est fait ethnocidaire, c'est par manque de pluralisme : il n'y a qu'une seule rationalité et elle est économique, donc chiffrable. Dès lors, des «mesures »- sorte de lapsus politique renfermant à la fois l'idée de la réforme et celle de la quantification - organisent l'élimination des paysans. Parmi elles, se trouvent la SMI (Surface minimum d'installation), qui conditionne l'accès aux aides en ne laissant subsister que les fermes jugées assez grandes pour être viables, et l'IvD (Indemnité viagère de départ), délivrée aux paysans de plus de 55 ans pour qu'ils cessent leur activité, libérant ainsi des parts de marché et des terres cultivables. Entre 1965 et 1985, ce sont plus de 11 millions d'hectares, soit $35 \%$ de la surface agricole utile, qui ont été « libérés » par l'IvD (p. 169). Dès 1945, des aides de l'État encouragent l'investissement en vue de l'agrandissement des exploitations et de l'augmentation de la production agricole. Le vivant, pour être exporté, doit être exploité industriellement et la science agronomique, à l'INRA (Institut national de recherche agronomique), se charge de créer des espèces animales et végétales plus productives. Les conséquences de ces mutations sont les crises régulières de surproduction et la diminution rapide et massive du nombre des paysans, qui engendre une baisse de la solidarité entre pairs.

En dissociant la ferme et l'entreprise, les syndicats majoritaires, FNSEA et $\mathrm{C}_{\mathrm{NJA}}{ }^{6}$, ont présidé à la gestion sociale de l'exode agricole. Pierre Bitoun et Yves Dupont leur opposent le développement de la gauche paysanne, notamment avec la création de Via Campesina, le mouvement international pour l'agriculture paysanne. Bien que minoritaire en France avec la Confédération paysanne, cette contestation politique du progrès technico-économique parvient néanmoins à mobiliser le répertoire critique du " traditionalisme de résistance ", conceptualisé par Georges Balandier.

9 À défaut de révéler des faits nouveaux, cet essai parvient à réinscrire la disparition des paysans dans une historicité complexe et à la montrer pour ce qu'elle est, à savoir un bouleversement majeur du $\mathrm{Xx}^{\mathrm{e}}$ siècle qui n'a de cesse de retentir dans notre présent. La tonalité critique, parfois amère, avec laquelle est scandée cette histoire gagnerait toutefois à préciser ses concepts pour mieux atteindre les phénomènes qu'elle attaque, à l'instar du «capitalisme industrialo-financier allié à la technoscience » (p. 230) comme figure de la «modernité ». Les typologies, parfois rapides, peuvent laisser songeur quant à leurs critères sélectifs, par exemple ces « cinq Eurêka de l'Archimède moyen»(p. 139) qui juxtaposent djihadisme, populisme d'extrême droite, convivialisme, simplicité volontaire et nomadisme connecté. Toutes ces attitudes sont perçues par Pierre Bitoun et Yves Dupont comme des comportements religieux, si ce n'est syncrétiques, qu'adoptent des individus pour pallier l'absurdité de leur existence et du monde qu'ils habitent. À ce titre, le choix du terme éponyme de sacrifice, qui charrie tant et tant de représentations sacrées et religieuses, n'est pas rendu explicite, alors que celui d'ethnocide, abondamment mobilisé dans le corps du texte, exprimerait au plus près le processus décrit par les auteurs.

10 Enfin, ces critiques n'éclipsent en aucun cas l'intérêt incontestable du Sacrifice des paysans, qui procure à ses lecteurs des outils historiques nécessaires et utiles pour comprendre les racines de la « crise » agricole contemporaine. 


\section{NOTES}

1. Pierre Alphandéry, Pierre Bitoun \& Yves Dupont, Les Champs du départ. Une France rurale sans paysans?, Paris, La Découverte, 1989 («Cahiers libres »).

2. Pierre Alphandéry, Pierre Bitoun \& Yves Dupont, L'Équivoque écologique, Paris, La Découverte, 1989 ( Cahiers libres »).

3. Michel Gervais, Claude Servolin \& Jean Weil, Une France sans paysans, Paris, Le Seuil, 1965 («Société » 7) ; Henri Mendras, La Fin des paysans, suivi d'une réflexion sur la fin des paysans, Le Paradou, Actes Sud, 1984 ; Bertrand Hervieu \& Jean Viard, L'Archipel paysan. La fin de la république agricole, La Tour d'Aigues, Éd. de l'Aube, 2005.

4. «La dépréciation du monde des hommes augmente en raison directe de la mise en valeur du monde des choses » (Karl Marx, Manuscrits de 1844, Paris, Éd. sociales, 1972 : 57).

5. Cf. Alain Supiot, La Gouvernance par les nombres. Cours au Collège de France, 2012-2014, Nantes, Institut d'études avancées de Nantes / Paris, Fayard, 2015 (« Poids et mesures du monde »).

6. Respectivement : la Fédération nationale des syndicats d'exploitants agricoles et le Centre national des jeunes agriculteurs, renommé Jeunes agriculteurs (JA) en 2002. Les deux syndicats sont très proches.

7. Georges Balandier, Le Détour. Pouvoir et modernité, Paris, Fayard, 1985 («L'Espace du politique ») : 167 . 